\begin{tabular}{|l|l|l|l|l|}
\hline Revista Clío América & ISSN: 1909-941X & Vol.9 & No. 17 & Enero - Junio de 2015 \\
\hline
\end{tabular}

\title{
Causas de desescolarización en las comunas 5, 6, 8 y 9 de Santa Marta (Colombia)
}

\author{
Causes of unschooling in the communes 5, 6, 8 and 9 of Santa Marta (Colombia)
}

Resumen: Este trabajo tiene el objetivo de conocer las principales causas de la desescolarización y su incidencia por sexo y edad en las comunas 5, 6, 8 y 9 del Distrito de Santa Marta en el año 2011. Para cumplir este objetivo se aplicó un censo en el área de estudio, se realizó una caracterización de los desescolarizados y se aplicó un modelo de regresión logística para identificar las variables que inciden en la probabilidad de ser desescolarizado. Entre los resultados encontrados, las razones que más se presentan como causa de la desescolarización son los problemas en la casa, el embarazo, la maternidad o paternidad, la necesidad de trabajar, el cambio de casa de la familia, entre otros presentados en este trabajo. Finalmente, estos resultados permiten señalar la necesidad de desarrollar estrategias enfocadas a mitigar el impacto de la desescolarización en los distintos grupos de edad y de manera diferencial para cada uno de los sexos.

Palabras Clave: Desescolarizado, causas de desescolarización, matrícula escolar, inasistencia escolar, tasa de asistencia escolar, falta de cupos escolares, comuna.

JEL: I20

\begin{abstract}
This paper has the objective to identify the main causes of unschooling and its incidence by sex, age in the commune 5, 6, 8 and 9 of the District of Santa Marta in 2011. To meet this objective a census was applied in the study area, a characterization of unschooling was conducted and a logistic regression model was applied to identify the variables that affect the probability of being unschooled. Found among the reasons most frequently presented as a cause of unschooling are the problems at home, pregnancy, parenthood, the need to work, changing family home, among others presented in this paper. Finally, these results allow to point out the need to develop strategies aimed at mitigating the impact of unschooling in different age groups and differentially for each of the sexes.
\end{abstract}

KEYWORDS: Unschooled, causes of unschooling, school enrollment, truancy, school attendance rates, lack of school places, commune.

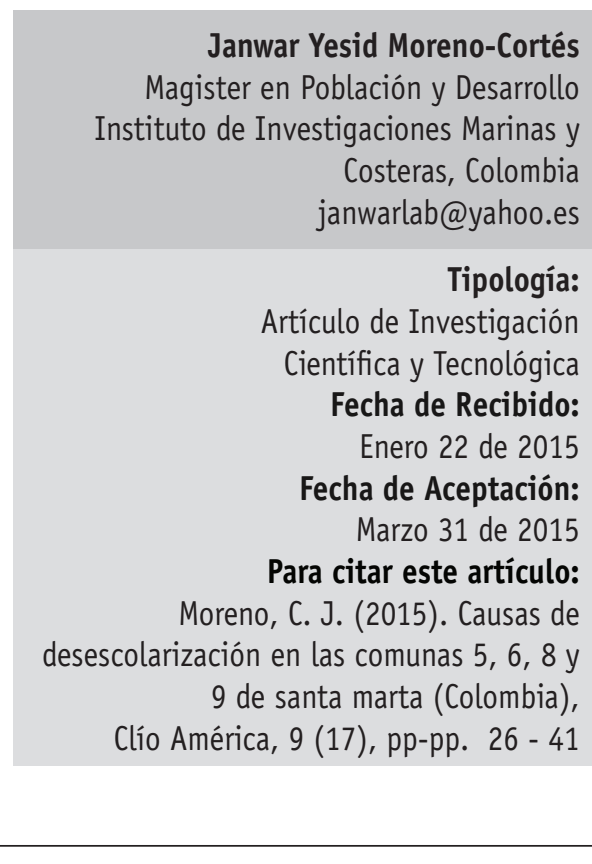

Clío América. Enero - Junio 2015, Vol. 9 No. 17, p.p. 26 - 41 


\section{Introducción}

La educación es analizada desde la economía como una inversión que constituye un aporte importante al desarrollo económico. Al analizar su impacto se busca maximizar la rentabilidad social de la inversión en educación, de este análisis se señalan los efectos de la educación, que se pueden distinguir analíticamente en: i.) el aumento de la productividad del trabajo; ii.) el crecimiento del producto nacional (beneficio para el país) y del ingreso (beneficio para el individuo); iii.) disminución del tiempo para el acceso al mercado laboral, que beneficia al individuo y a la sociedad (Cohen, 1997, p. 7). Este enfoque de análisis resalta la importancia de la educación para el desarrollo de los individuos y el desarrollo nacional, indicando que la tasa de rentabilidad del sector educación básica alcanzó en el mundo el 20\% entre 1974 y 1992, por encima de tasas de rentabilidad en sectores como la agricultura, la industria y la infraestructura (BANCO MUNDIAL, 1995).

Ante la importancia de la educación por su rentabilidad, este se constituye en un eje de vital importancia para el desarrollo humano, el cual es incluido en los objetivos de desarrollo del milenio, que plantea como objetivo lograr la enseñanza primaria universal (teniendo en cuenta que es en la primaria donde se genera la mayor rentabilidad social, según el Banco Mundial (1995)), para el caso colombiano se contempla el derecho a la educación en el artículo 67 de la Constitución Política de 1991, definiéndola como "un derecho de la persona y un servicio público que tiene una función social" (Constitución Política de Colombia, 1991), este principio señala que es responsabilidad del Estado velar por el adecuado cumplimiento del servicio.

Uno de los principales problemas en el tema de educación, es la desescolarizaión, que no permite a algunos estudiantes terminar el ciclo de formación. El joven desescolarizado, desde una perspectiva interaccionista puede ser considerado como un "desviante escolar", en donde la persona desde el punto de vista del grupo, ha transgredido una norma, y que además puede ocasionar un efecto negativo sobre la sociedad; si se analiza como un problema vinculado al orden y se relaciona en un contexto de alto desempleo, surgen interrogantes sociales como: “¿qué pasa con los jóvenes fuera de la escuela?, ¿están en riesgo de delincuencia?, ¿están expuestos a diversos tráficos o vagando por la calles sin protección?". (Becker, 1985; citado en Esterle, 2005, p. 33).

Entre los factores causantes de la desescolarización se encuentran: el nivel socioeconómico de las familias, y en los primeros grados la indisciplina y la búsqueda de identidad (Esterle, 2005). Otros estudios señalan otras causas de la desescolarización, entre ellas tenemos: las causas centradas en el entorno, causas centradas en la escuela, las causas centradas en la familia, y las causas centradas en el individuo; los dos últimos mencionados también por Esterle, (2005); (GOBIERNO VASCO, 2003).

En América Latina existen unos temas emergentes que dificultarían la universalización de la educación primaria y estimulan la deserción escolar, como son: los riesgos naturales y conflictos sociales, necesidades educativas especiales, falta de inclusión y la educación bilingüe intercultural, así como es necesario tener en cuenta el acceso y la supervivencia al sistema educativo en los distintos niveles, principalmente en Colombia, Dominica, El salvador, Granada, Islas Turcas y Caicos, Nicaragua, República Dominicana y República Bolivariana de Venezuela. Es de destacar que en América Latina los hombres acceden a la educación secundaria en menor proporción que las mujeres, o abandonan la escuela antes, situación explicada en parte porque los jóvenes, principalmente de bajos estratos, realizan a una edad temprana actividades remuneradas (UNESCO, CEPAL, 2005).

De igual forma, para el caso de América Latina se observa un desfase entre las características de la educación y la demanda laboral, que conlleva a dificultar el canal de la movilidad social agravando la desigualdad en la distribución del ingreso en la región, este desfase se manifiesta de manera más elevada y prolongada en los jóvenes, debido a niveles inferiores de educación y de habilidades adquiridas (Weller, 2009, p. 15).

En el caso colombiano, a partir de 1986 la tendencia en las variables educativas cambia positivamente, producto del esfuerzo intencional y de los gobiernos 
del momento por aumentar los matriculados, principalmente en educación primaria; este proceso sucede en el marco de la descentralización del sector educativo que inicia formalmente en 1986, además que lleva a fortalecer el marco de la nueva Constitución Política de Colombia de 1991, Vélez (2003) citado en Ramírez \& Téllez (2006) señala que “para finales de siglo XX el $12 \%$ de los niños en edad escolar en Colombia no pueden asistir a la educación primaria, disminuyendo considerablemente con respecto a los inicios de los años 80, sin embargo, esta situación contrasta con el $9 \%$ en el resto de América Latina" (p. 66), señalando que aún el país se encontraba con indicadores por encima del promedio de América Latina.

Enfocándonos en el Distrito de Santa Marta, para el año 2005 según datos del censo de ese año, habían 114.657 personas que no asistían a la escuela, la mayor razón de inasistencia a la escuela fue la necesidad de trabajar (29.285 personas); también resulta considerable el número de personas que manifiesta no asistir a la escuela por los altos costos educativos (28.946 personas); el 7,1\% no asiste a la escuela porque considera que ya terminó sus estudios; $4,9 \%$ por falta de cupos; 3,9\% por la lejanía del establecimiento; una razón relevante es el embarazo que alcanza el $2,9 \%$ de no asistencia a la escuela por esta causa, la relevancia de la variable embarazo es su efecto en el nivel de pobreza (Tabla 1).

\section{Tabla 1.}

\section{Número de personas por razones de inasistencia a la escuela en la ciudad de Santa Marta (2005)}

\begin{tabular}{|c|c|c|}
\hline $\begin{array}{c}\text { Razones de inasistencia } \\
\text { a la escuela }\end{array}$ & Personas & Porcentaje \\
\hline $\begin{array}{c}\text { Otra causa de } \\
\text { inasistencia }\end{array}$ & 32.860 & 28,7 \\
\hline Costos educativos altos & 28.946 & 25,3 \\
\hline Necesita trabajar & 29.295 & 25,6 \\
\hline Falta de cupos & 5.583 & 4,9 \\
\hline $\begin{array}{c}\text { Lejanía del } \\
\text { establecimiento }\end{array}$ & 4.463 & 3,9 \\
\hline
\end{tabular}

\begin{tabular}{|c|c|c|}
\hline $\begin{array}{c}\text { Razones de inasistencia } \\
\text { a la escuela }\end{array}$ & Personas & Porcentaje \\
\hline Enfermedad & 1.861 & 1,6 \\
\hline Embarazo & 3.293 & 2,9 \\
\hline $\begin{array}{c}\text { Considera que ya } \\
\text { terminó estudios }\end{array}$ & 8.154 & 7,1 \\
\hline Total & 114.457 & 100 \\
\hline
\end{tabular}

Fuente: Estadísticas del DANE, Censo 2005.

Como punto de comparación entre los demás municipios del departamento del Magdalena y la situación del Distrito de Santa Marta, se presenta la tasa de asistencia escolar, donde se observa que en comparación con los demás municipios, Santa Marta presenta la mayor tasa de asistencia escolar (entre $80,9 \%$ y $87,5 \%$ ), al igual que los municipios de Salamina, Cerro de San Antonio, San Sebastián de Buenavista y Guamal. En cuanto al entorno (municipios vecinos) todos presentan una tasa de asistencia escolar menor, resalta la baja asistencia escolar en Aracataca que se encuentra entre $62,1 \%$ y $73,4 \%$ ( Figura 1).

Uno de los principales problemas que enfrenta el Distrito de Santa Marta es la dificultad para identificar y caracterizar la población que se encuentra por fuera del sistema escolar, esta carencia imposibilita que se diseñen y establezcan políticas públicas que direccionen y fortalezcan el sistema educativo, generando dificultades para el acceso a una oferta de educativa oportuna y pertinente, que garantice la permanencia y la calidad educativa para los niños, niñas, jóvenes y adultos.

A partir de la necesidad de información para la toma de decisiones, surge esta investigación que busca resolver la siguiente pregunta: ¿Cuáles son las principales causas de desescolarización en las comunas 5, 6, 8 y 9 de Santa Marta?, resolver esta pregunta es importante porque permite plantear estrategias coherentes con el contexto social de estas comunas de Santa Marta y a las características del habitante. Para resolver esta pregunta, se diseñó y aplicó el censo educativo en las comunas 5, 6, 8 y 
9 de esta ciudad, que consistió en la aplicación de un cuestionario vivienda por vivienda a todos los habitantes de las comunas analizadas, información que fue digitalizada y analizada, además se estima un modelo logístico que indaga sobre las principales causas de la desescolarización.

\section{- Figura 1.}

Tasa de asistencia escolar en edad de 3 a 17 años en los municipios del Magdalena (2005)

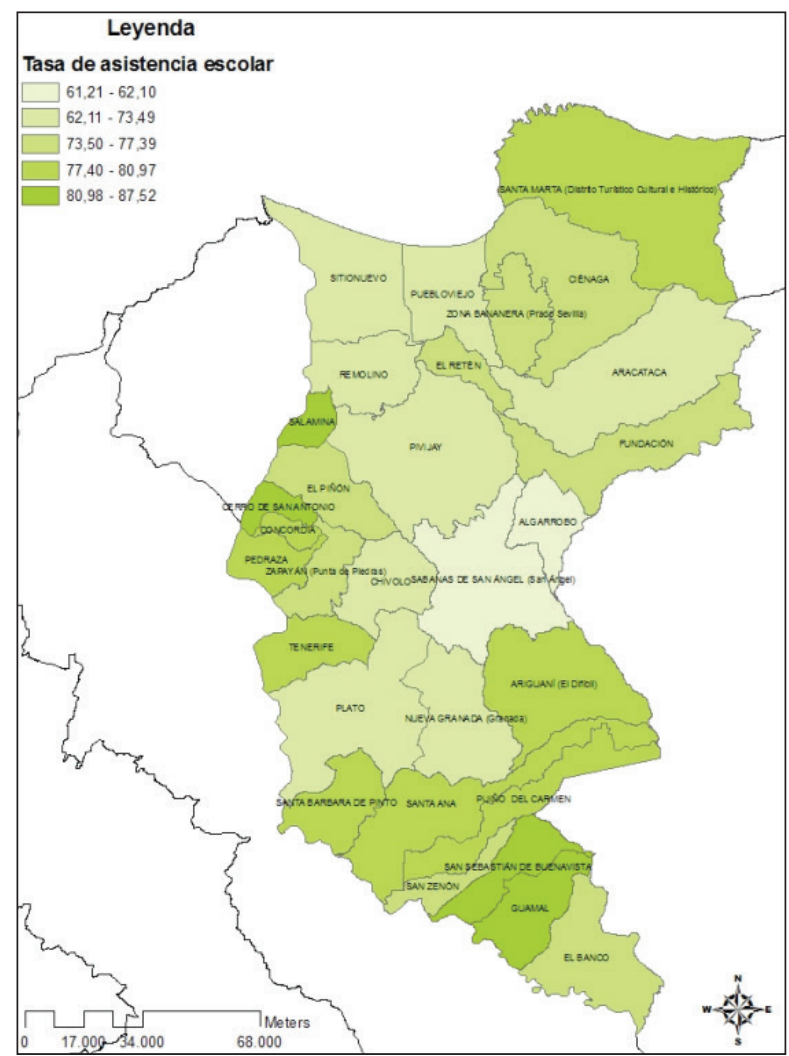

Fuente: Elaboración propia basada en información de cobertura geográfica del SIGOT (2005)

\section{Metodología}

La metodología de este trabajo está enfocada en la identificación de hogares que principalmente presentan desescolarizados, en ese sentido se realizó un censo en las viviendas de las comunas 5, 6, 8 y 9 de Santa Marta, el censo es un ejercicio en el que se aplica un cuestionario a toda la población de estas comunas. El proceso metodológico y operativo de la operación censal se desarrolló en cinco (5) fases descritas de la siguiente manera; Fase 1: Planeación y Conformación del equipo operativo y técnico del Censo; fase 2: Capacitación e instrucción al equipo operativo y técnico del censo; fase 3: Recuento de manzanas y viviendas; fase 4: Aplicación de las encuestas; fase 5: Sistematización, Procesamiento y Análisis de la información (Tabla 2).

\section{Tabla 2.}

\section{Resumen de la Ficha técnica del Estudio Censo Desescolarizados entre 5 y 24 años de las Comunas 5, 6, 8 y 9 del Distrito de Santa Marta (2011)}

\begin{tabular}{l|c|}
\hline Departamento & Magdalena \\
\hline Municipio & Santa Marta \\
\hline Comunas & 31 \\
\hline Barrios & y 9 \\
\hline Instrumento aplicado & $\begin{array}{c}\text { Encuesta casa a casa } \\
\text { Toda los residentes } \\
\text { permanentes en los } \\
\text { barrios objeto de } \\
\text { estudio }\end{array}$ \\
\hline Población & 11.113 \\
\hline $\begin{array}{l}\text { Total de Hogares } \\
\text { encuestados }\end{array}$ & 29.779 \\
\hline $\begin{array}{l}\text { Total personas } \\
\text { encuestadas }\end{array}$ & 11.028 \\
\hline $\begin{array}{l}\text { Total personas entre } \\
5 \text { y } 24 \text { años }\end{array}$ & 1.716 \\
\hline $\begin{array}{l}\text { Total personas en- } \\
\text { tre } 5 \text { y 24 años } \\
\text { desescolarizados }\end{array}$ & 23.868 \\
\hline $\begin{array}{l}\text { Total datos (perso- } \\
\text { nas) validos (datos } \\
\text { que incluyen todas } \\
\text { las variables) }\end{array}$ & \begin{tabular}{l} 
a \\
\hline
\end{tabular}
\end{tabular}

Fuente: Censo desescolarizados Comunas 5, 6, 8 y 9 


\section{Resultados}

Los resultados generales del censo de desescolarizados, nos permiten observar el número de personas y su porcentaje según su condición educativa, de este modo tenemos que la población en el rango de edad entre 5 y 24 años equivale al $37 \%$ de la población total del censo, de ese grupo poblacional el $80 \%$ no es bachiller, el $20 \%$ restante es bachiller de los cuales la mayoría no asiste a un centro educativo (58\%). Los no bachilleres y que no asisten a un centro educativo son precisamente el grupo poblacional que para efectos de este trabajo son denominados los desescolarizados, que ascienden a 1.716 y representan el $20 \%$ de las personas que no son bachilleres. A continuación se realizará un análisis especificando las características de los desescolarizados (Figura 2).

HFigura 2.

Resultados generales del censo de desescolarizados de las Comunas 5, 6, 8 y 9 del Distrito de Santa Marta (2011)

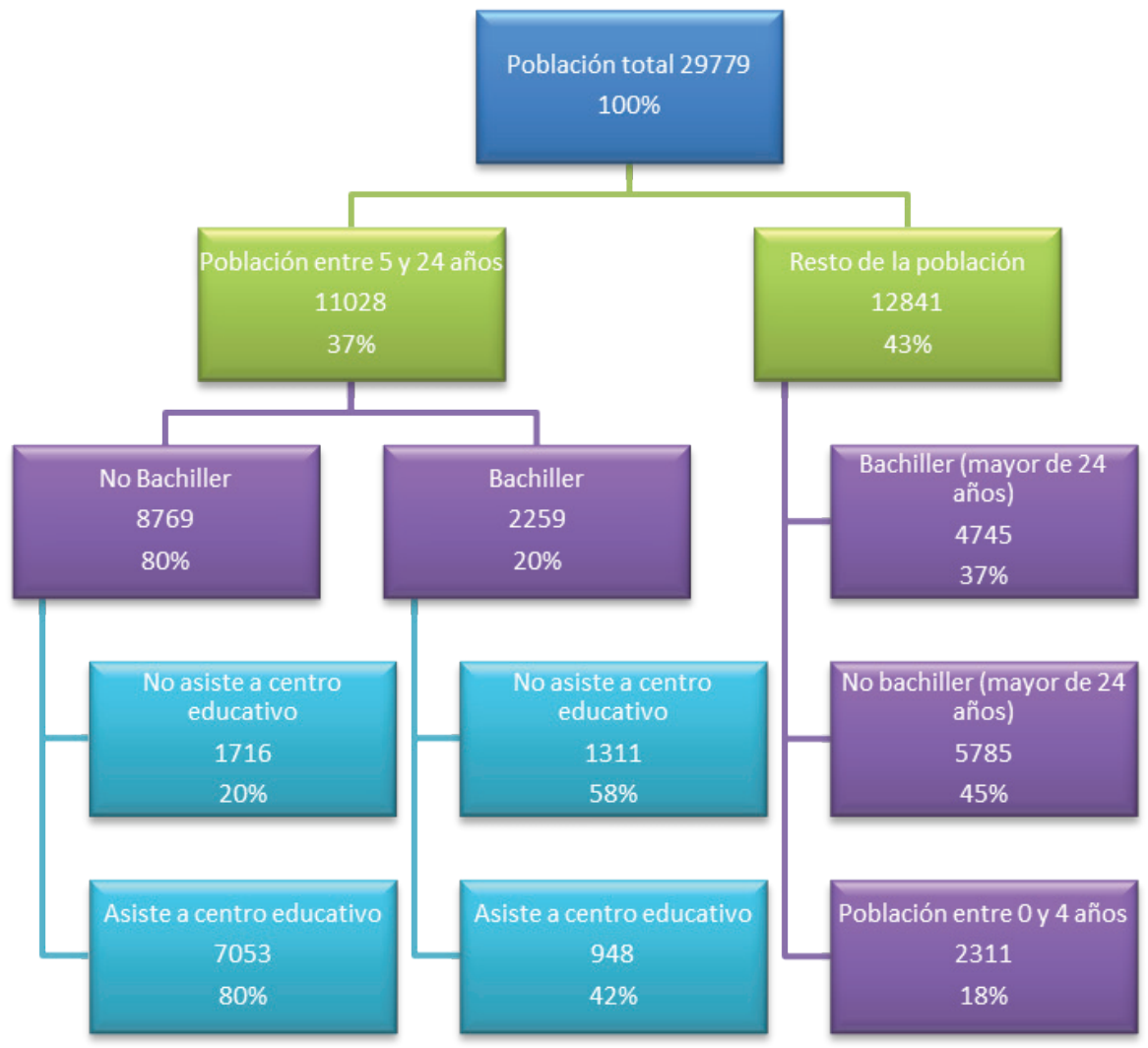

Fuente: Elaboración propia basado en la información del censo de desescolarizados. 
Por otra parte, se pueden observar algunas características relevantes en aquellas personas que no se encuentran en la edad de 5 y 24 años, que en la Figura 2 se denominan "resto de la población", el grupo de personas con edad mayor a 24 años y que no son bachilleres son 5.785 personas que alcanza el $45 \%$ del resto de la población, solo el $37 \%$ es bachiller, y el restante $18 \%$ son menores de 5 años. Esta información resulta de importancia si se tiene en cuenta el bajo nivel de formación que tiene un gran porcentaje de población, que aunque no se encuentra en el rango de edad entre 5 y 24 años, no ha tenido ni tiene acceso al sistema educativo para poder dar por terminados sus estudios hasta el bachillerato.

\section{Características de la población desescolarizada}

A continuación se presentan un grupo de características asociadas a la población desescolarizada en el año 2011, subdividiendo este componente en: i) características asociadas al hogar; y ii) características asociadas directamente con el individuo desescolarizado. E esta última característica se presenta el patrón de la desescolarización y sus diferencias por sexo y edad, así como la incidencia del fenómeno en las comunas.

\section{Características del hogar}

Ingreso mensual: la primera característica de observación es el ingreso mensual de los hogares desescolarizados, para lo cual se encuentra que el mayor grupo de población $(33,5 \%)$ recibe entre $\$ 400.000$ y $\$ 600.000$, seguido por el $31,2 \%$ de la población que recibe entre $\$ 100.000$ y $\$ 400.000$, es así como se observa que más del $60 \%$ de la población recibe entre $\$ 100.000$ y $\$ 400.000$ pesos, por encima de este rango de ingreso (reciben más de $\$ 600.000$ ) se encuentra más del $15 \%$, y entre 0 y $\$ 100.000$ el $7,7 \%$ (Figura 3).
- Figura 3.

\section{Porcentaje de hogares con población desescolarizada por rango de ingresos}

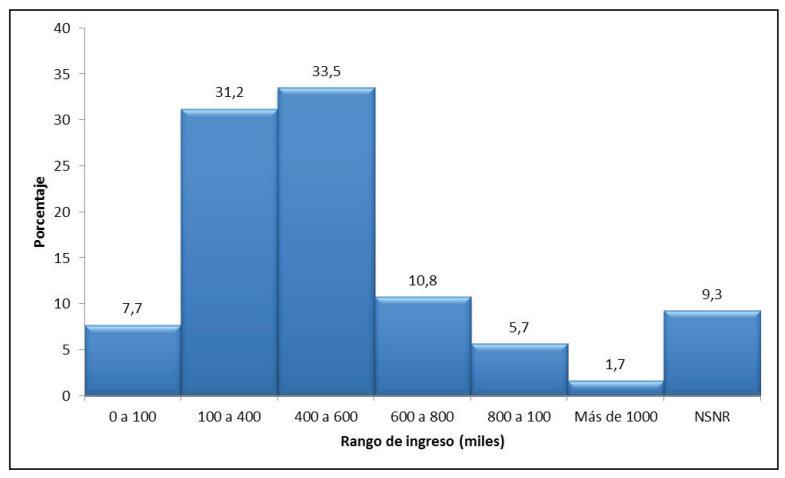

Fuente: Elaboración propia basado en la información del censo de desescolarizados.

\section{Desescolarizados y estrato socioeconómico del} hogar: el mayor porcentaje de hogares con personas desescolarizadas pertenecen al estrato uno (71\%), señalando una relación entre estrato socioeconómico bajo y desescolarización, en el estrato dos se encuentra el $9,1 \%$, y solo el $0,4 \%$ en el estrato tres, este último es el mayor estrato socioeconómico alcanzado por un hogar desescolarizado (Figura 4)

\section{Figura 4.}

\section{Porcentaje de hogares con población desescolarizada por estrato socioeconómico}

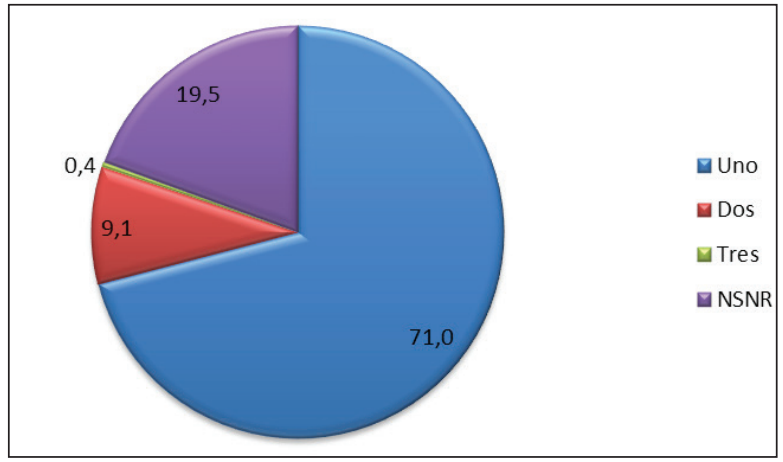

Fuente: Elaboración propia basado en la información del censo de desescolarizados. 
Desescolarizados y servicios públicos en la vivienda: para analizar las condiciones de la vivienda se presenta la característica de servicios públicos con los que cuenta la vivienda.

\section{Figura 5.}

\section{Porcentaje de hogares con desescolarizados por servicios públicos}

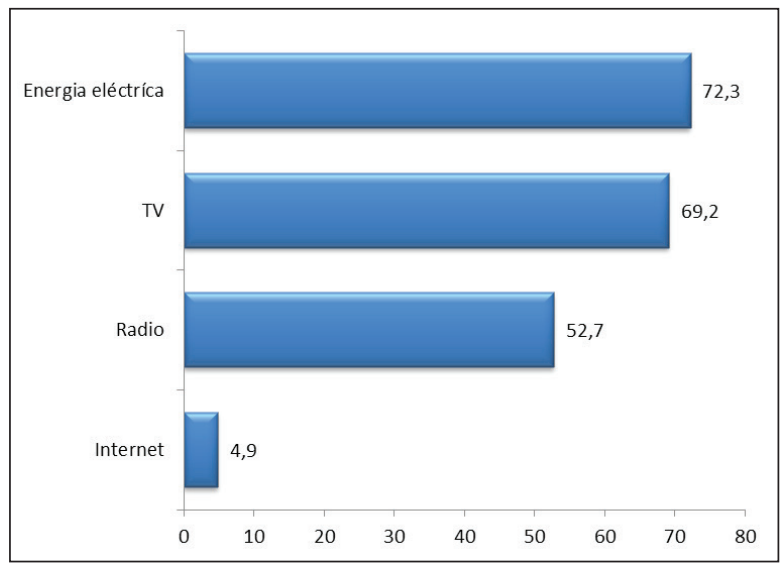

Fuente: Elaboración propia basado en la información del censo de desescolarizados.

Se observa que la gran mayoría de hogares (más del $50 \%$ ) cuenta con el servicio de radio, televisión (TV) y energía eléctrica en su casa, siendo la última la de mayor cobertura $(72,3 \%)$, pero aún existe un porcentaje de la población desescolarizada que no tiene estos servicios en el hogar. Por otra parte, la cobertura en el servicio de internet resulta bastante baja, puesto que solo el $4,9 \%$ de la población tiene internet en su casa, esta puede presentarse como una desventaja en la consolidación de procesos formativos de capital humano en donde se utilicen las tecnologías de la información y la comunicación (TIC) (Figura 5).

\section{Desescolarizados afectados por una situación de} violencia: el grupo de población desescolarizada pueden ser afectados con mayor incidencia por una situación de violencia, si se tiene en cuenta el contexto social y la marginalidad en que se pueden encontrar esta población. La información del censo permite observar que más del $50 \%$ de la población ha sufrido alguna situación de violencia, de manera precisa solo el $42,1 \%$ manifiesta no haber sufrido ninguna situación de violencia (Figura 6).

Figura 6.

\section{Porcentaje de hogares con población desescolarizada por situación de violencia}

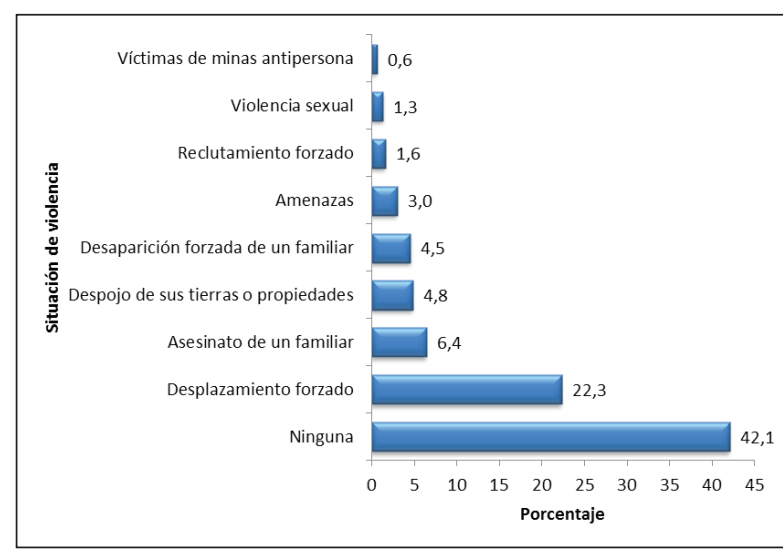

Fuente: Elaboración propia basado en la información del censo de desescolarizados.

La situación de violencia con más frecuencia entre la población desescolarizada es el desplazamiento forzado (el 22,3\% de la población lo ha sufrido), este presenta una distancia significativa con las demás situaciones, seguido de la situación del asesinato de un familiar con el $6,4 \%$, el despojo de tierras el $4,8 \%$, la desaparición forzada de un familiar con el $4,5 \%$, las amenazas con el $3 \%$, el reclutamiento forzado con el $1,6 \%$, la violencia sexual el $1,3 \%$, y el $0,6 \%$ manifiesta haber sido víctima de las minas antipersona (Figura 6). En este contexto, resulta importante resaltar la gran magnitud del desplazamiento forzado en los desescolarizados, puesto que esta situación puede incidir en el direccionamiento de las estrategias para enfrentar la desescolarización.

Desescolarizados y ayudas recibidas por el gobierno: en los hogares con personas desescolarizadas se observa que el $43,6 \%$ de la población manifiesta no haber recibido ayudas del gobierno, sin embargo es considerable que el $27,7 \%$ de la población ha recibido ayudas del programa "Familias en acción", 
es a partir de esta ayuda que se canalizan el mayor número de recursos para los hogares con población desescolarizada, también se observa con mayor frecuencia ayudas de la "Red Juntos" $(2,3 \%)$, ayuda humanitaria de emergencia con el $1,7 \%$, mercados de la alcaldía con el $0,8 \%$ y algún otra ayuda el 0,9\% (Figura 7).

\section{- Figura 7.}

\section{Porcentaje de hogares con desescolarizados por ayudas recibidas del gobierno}

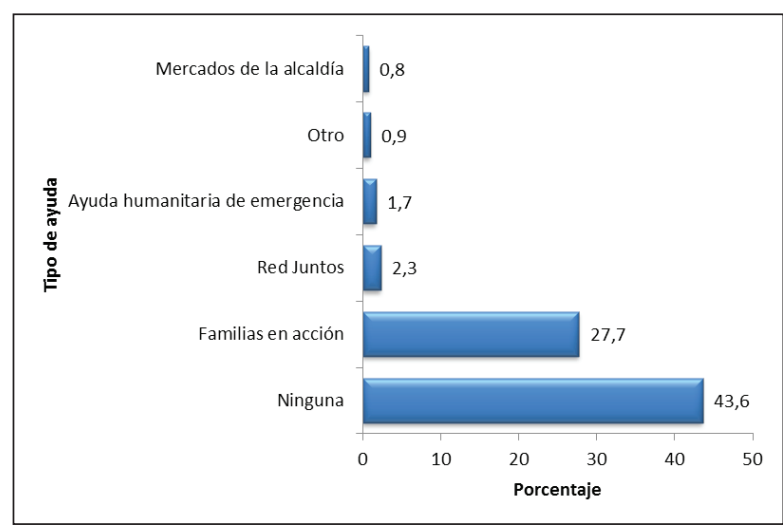

Fuente: Elaboración propia basado en la información del censo de desescolarizados.

Como una forma de indagar sobre las posibles causas de la situación de desescolarización del individuo, se preguntó sobre el tiempo necesario para llegar a la escuela más cercana que conocía el grupo familiar, de este modo, se observa que en su mayoría $(88 \%)$ las personas gastarían menos de 15 minutos en llegar a la escuela, solo el 9,5\% entre 16 a 30 minutos, de 31 a 60 minutos el 2,3\% y más de 60 minutos el $0,2 \%$ (Figura 8 ). De este modo, se observa que el problema del tiempo de desplazamiento entre la escuela y la vivienda, no se consolida como un problema importante, puesto que la mayoría de población gasta menos de 15 minutos en llegar a la escuela.
Figura 8.

\section{Porcentaje de hogares con población desescolarizada por tiempo de desplazamiento entre la escuela y la vivienda}

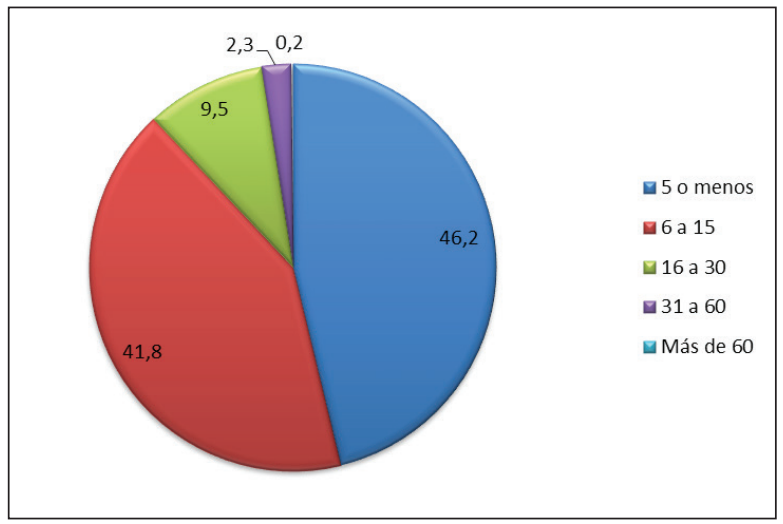

Fuente: Elaboración propia basado en la información del censo de desescolarizados.

\section{Características personales}

Desescolarizados por sexo y edad: el comportamiento de la desescolarización por sexo y edad nos permite observar que la desescolarización se presenta con mayor intensidad en las personas con edad de 5 años (4\% para hombre y 3,7\% para las mujeres) y de los 17 años en adelante (Figura 9).

Figura 9.

\section{Porcentaje de desescolarización por sexo y edad}

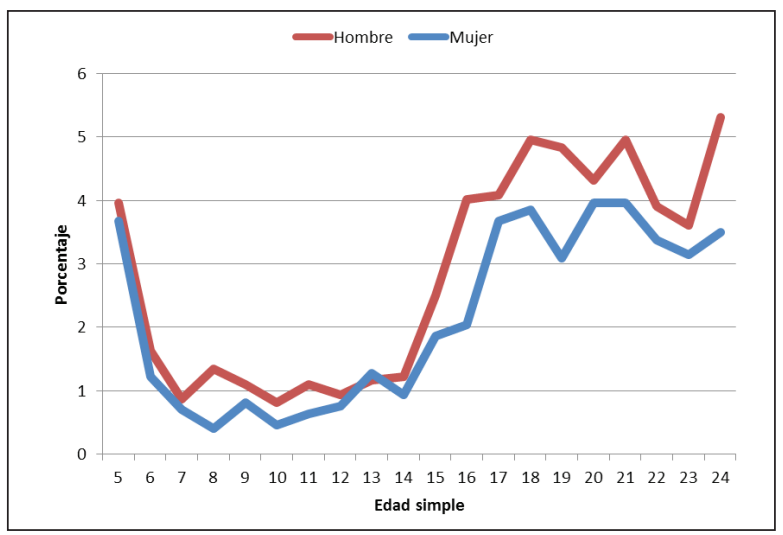

Fuente: Elaboración propia basado en la información del censo de desescolarizados. 
De este modo podemos observar un comportamiento parabólico, en donde los puntos de menor incidencia de la desescolarización se encuentran en la edad entre 7 y 14 años, esta relación de mayor incidencia de la desescolarización en las edades mencionadas puede entenderse porque a la edad de 5 años muchos niños aún no son matriculados, retrasando su ingreso a la escuela, y de los de 17 años en adelante se pueden relacionar con presiones para la realización de actividades laborales para llevar sustento a la familia. Con relación al sexo se puede observar que para todas las edades existe una mayor incidencia de la desescolarización en los hombres, denotándose con mayor perseverancia en los rangos de edad mayores a los 15 años, solo a la edad de 13 años es mayor la desescolarización en las mujeres (Figura 9)

Desescolarizados por comuna y sexo: los desescolarizados se ubican en su mayoría en la comuna 6 (Mamatoco-11 de Noviembre), alcanzando los 967 desescolarizados, seguido por la comuna 8 (Pozos Colorados-Don Jaca) que alcanza los 202 desescolarizados, en la comuna 9 (Parque-Bureche) se observan 253 desescolarizados, y en la comuna 5 (Santa Fe-Bastidas) se encuentran 59 desescolarizados; en cuanto al sexo la incidencia de la desescolarización es mayor en los hombres, en valores absolutos la diferencia es mayor en la comuna Mamatoco-11 de Noviembre, sin embargo, en valores relativos relacionado con el total de desescolarizados se presenta que en la comuna Parque-Bureche se tienen las mayores diferencias por sexo (Figura 10).

Desescolarizados por razón de inasistencia a la escuela: a continuación se presentan las razones de inasistencia que manifestaron los encuestados, están han sido agrupadas por importancia porcentual, de este modo se presentan las de mayor importancia porcentual (el criterio de agrupación fueron las mayores del $3 \%)$, y se presentan a aquellas razones de menor importancia porcentual ${ }^{1}$. Esta información permitirá confirmar las razones que presentó la población para permanecer desescolarizada, permi-

1. Es preciso aclarar que las causas expuestas a continuación no son mutuamente excluyentes, es decir, una persona puede manifestar más de una causa de desescolarización, de este modo, los resultados porcentuales por razones se refieren al porcentaje que manifestó la causa especificada y que puede contestar afirmativamente a otra razón, así al no ser mutuamente excluyentes no se deben sumar los porcentajes en las razones de desescolarización. tiendo corroborar las hipótesis expuestas por otros estudios y que en este trabajo son mencionadas en el marco referencial.

Figura 10.

\section{Desescolarizados por comuna y sexo}

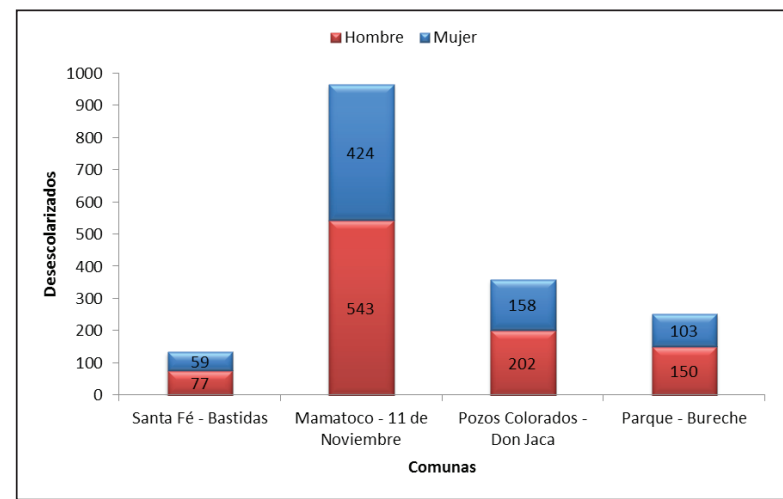

Fuente: Elaboración propia basado en la información del censo de desescolarizados.

fFigura 11.

\section{Razones de inasistencia a la escuela de mayor frecuencia}

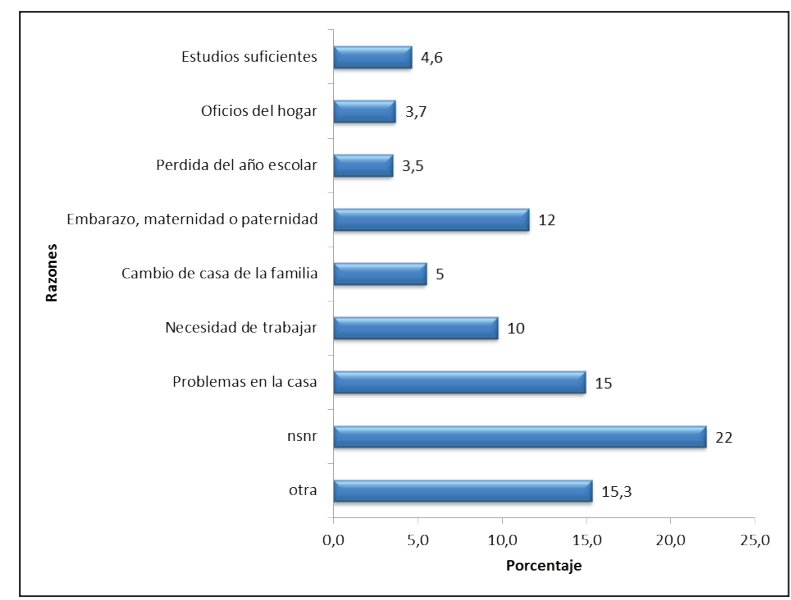

Fuente: Elaboración propia basado en la información del censo de desescolarizados.

En primera instancia, se observa que los problemas en la casa son la principal causa de desescolarización $(15 \%)$, seguido por el embarazo, maternidad 
0 paternidad $(12 \%)$, la necesidad de trabajar presenta importancia (10\%), el cambio de casa de la familia (5\%), porque considera que los estudios son suficientes $(4,6 \%)$, los oficios del hogar $(3,7 \%)$ y perdida del año escolar $(3,5 \%)$, las otras razones agrupan el $5,3 \%$, de este modo es relevante entender estas razones y su comportamiento diferencial entre hombres y mujeres, así como en las distintas edades (Figura 11).

Figura 12.

\section{Razones de inasistencia a la escuela de menor frecuencia}

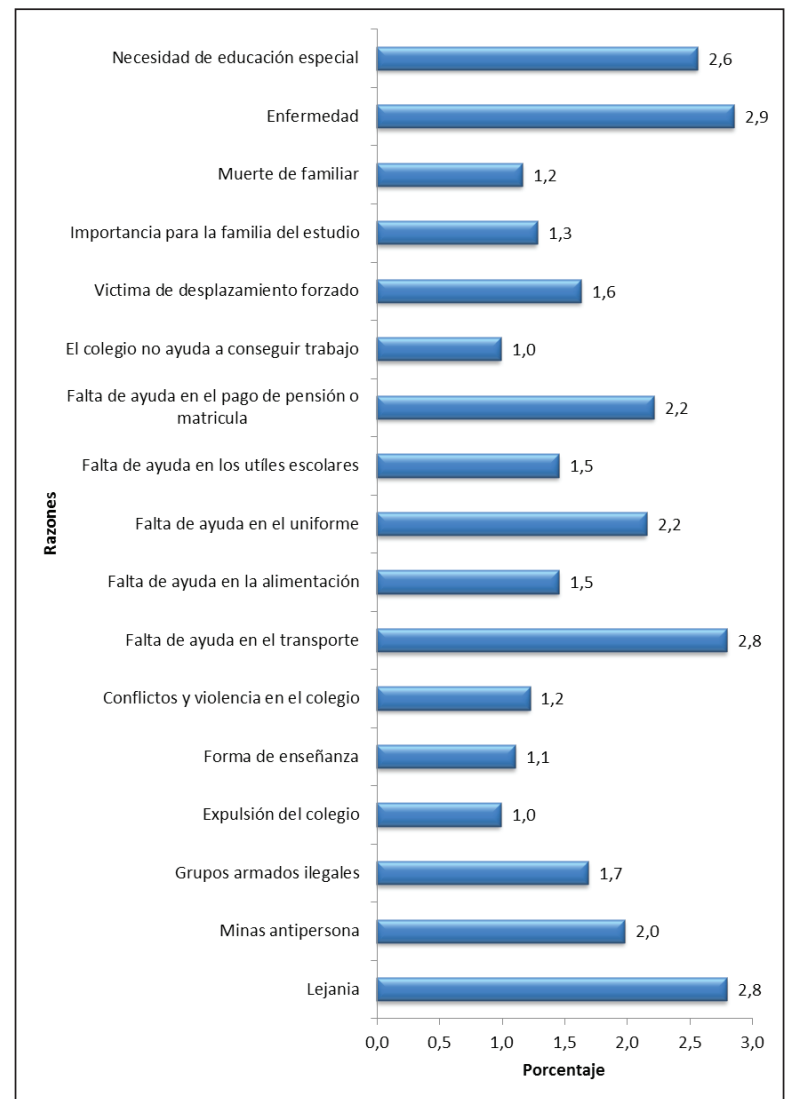

Fuente: Elaboración propia basado en la información del censo de desescolarizados.

Entre las razones con menor importancia porcentual se encuentran la enfermedad $(2,9 \%)$, la falta de ayuda en el transporte $(2,8 \%)$, la lejanía de la escuela $(2,8 \%)$, la necesidad de educación especial, y las otras relacionadas en la Figura 12.
Desescolarizados por problemas en la casa según sexo y edad: sobre la causa problemas en la casa se observa que esta se manifiesta con mayor trascendencia en los hombres, y en las edades de 15 a 24 años, alcanzando un máximo de 61 hombres que manifiestan esta razón; en las primeras edades la razón de problemas en la casa no se manifiesta con igual incidencia a las edades superiores, sentido contrario al manifestado por Esterle (2005) quien señala que en los primeros grados pueden existir niveles de desescolarización asociados a la búsqueda de identidad, para el caso de este trabajo se pueden identificar deserciones asociadas a problemas en la adolescencia, en donde los jóvenes están consolidando su identidad y expresando ideas de libertad con sus padres, de igual forma los problemas económicos en los hogares inciden en problemas que lleven al joven a salir de la escuela (Figura 13).

Figura 13.

\section{Desescolarizados por problemas en la casa según sexo y edad agrupada}

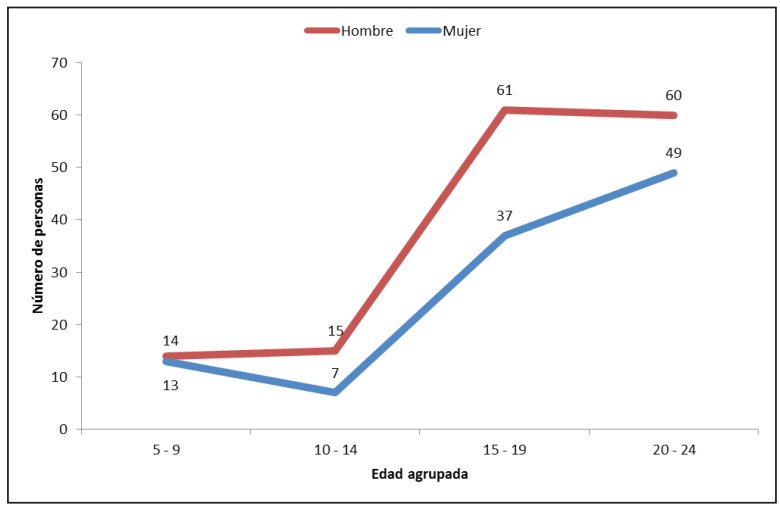

Fuente: Elaboración propia basado en la información del censo de desescolarizados.

\section{Desescolarizados por necesidad de trabajar según} sexo y edad: entre los 5 y los 14 años la necesidad de trabajar afecta de maneras similares a hombres y mujeres (Figura 14).

A partir de la edad de 15 años, aunque se observa una mayor afectación de la desescolarización por necesidad de trabajar para ambos sexos, en el caso de los hombres el efecto es mayor (alcanzando en 
los hombres el doble del valor absoluto de las mujeres), permitiendo de esta forma entender que la desescolarización por necesidad de trabajar es una de las causas que llevan a que principalmente los hombres no asistan a la escuela (Figura 14).

\section{Figura 14.}

\section{Desescolarizados por necesidad de trabajar según sexo y edad agrupada}

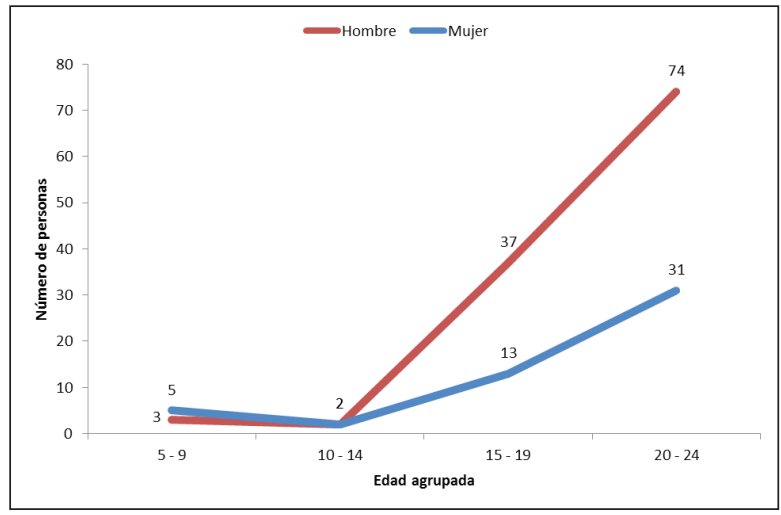

Fuente: Elaboración propia basado en la información del censo de desescolarizados.

Desescolarizados por embarazo, maternidad 0 paternidad según sexo y edad: la variable de razón de desescolarización por embarazo, maternidad 0 paternidad, cobra relevancia, tanto por su incidencia porcentual como también por el impacto social que genera esta variable, si se tiene en cuenta que el embarazo adolescente se presenta como una de las principales problemáticas en los jóvenes y en las escuelas, puesto que la aparición de este fenómeno limita las posibilidades de desarrollo personal; los jóvenes deben dedicarse a la crianza de hijos, cuando deberían estar en la escuela formándose para mejorar sus capacidades para ingresar al mercado laboral, y así mejorar su calidad de vida, por el contrario el embarazo adolescente lleva a robustecer las trampas de la pobreza, principalmente a las mujeres quienes son las que llevan el mayor peso de la crianza de los hijos.
De este modo, tal como se había mencionado, el efecto sobre la desescolarización es mayor en las mujeres, asociado al mayor peso en el periodo del embarazo y en las cargas del hijo. En cuanto al comportamiento de esta razón por edad se observa que el fenómeno se presenta con mayor incidencia en las mujeres en edad entre 15 a 19 años alcanzando a 77 mujeres, y que consecutivamente en la edad de 20 a 24 años disminuye a 67 casos. Este comportamiento permite percibir la edad con mayores problemáticas para las mujeres en cuanto a desescolarización y embarazo o maternidad, también es destacable en el sexo masculino un aumento de la incidencia de la desescolarización con la edad desde los 15 a los 24 años, mostrando que la paternidad también afecta a los hombres (en la edad de 5 a 9 años se observan dos casos masculinos y 5 femeninos que manifiestan la maternidad o paternidad como razón desescolarización, situación que físicamente es poco probable, por lo tanto debe referirse a que el encuestado respondió basado en la situación de un familiar que incide directamente en su decisión de estudiar) (Figura 15).

Figura 15.

\section{Desescolarizados por embarazo, maternidad 0 paternidad por sexo y edad}

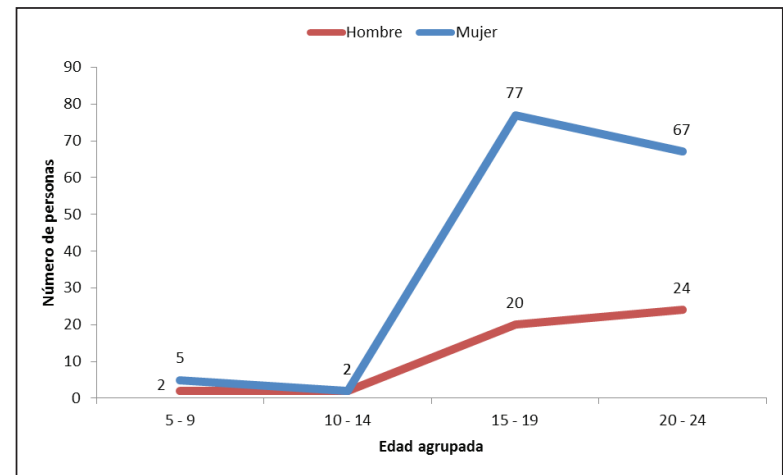

Fuente: Elaboración propia basado en la información del censo de desescolarizados. 
Desescolarizados por pérdida del año escolar según sexo y edad: en cuanto al componente de pérdida del año escolar se observa mayor incidencia en los hombres en todos los rangos de edad (Figura 16).

Figura 16.

\section{Desescolarizados por pérdida del año escolar según sexo y edad agrupada (2011)}

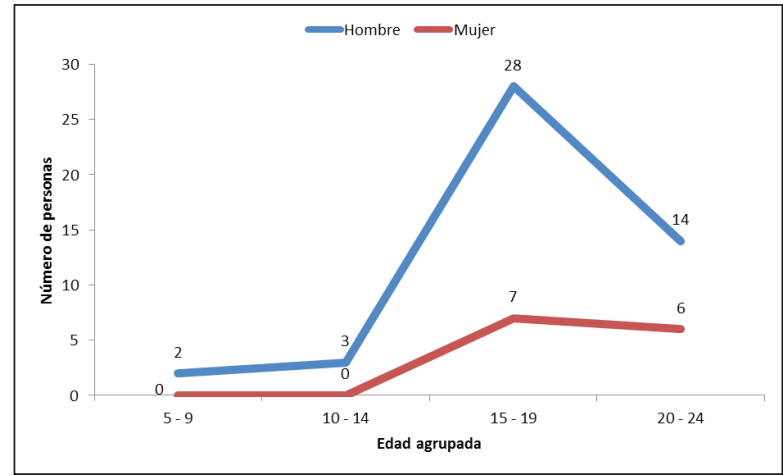

Fuente: Elaboración propia basado en la información del censo de desescolarizados.

De este modo de los 5 a los 14 años no se presentaron casos de mujeres que manifestaran la pérdida del año escolar como la razón de desescolarización; en la edad de 15 a 19 años se presenta la mayor diferencia entre sexos, puesto que mientras que hay 28 hombres desescolarizados por perdida del año escolar, en las mujeres solo alcanza a 7 casos, para la edad de 20 a 24 años la tendencia disminuye para los dos sexos, manteniéndose los hombres por encima a las mujeres (Figura 16).

Desescolarizados por realización de oficios en el hogar por sexo y edad: en cuanto a la razón manifiesta de desescolarización en donde la persona debe atender los oficios del hogar, se observa que el fenómeno de la desescolarización presenta mayor incidencia en los hombres en todos los rangos de edad, teniendo como punto máximo la edad de 15 a 19 años que alcanza 28 casos y que después en la edad de 20 a 24 años disminuye a 14 casos. Para el caso de las mujeres, de igual forma, el mayor número de casos se observa en la edad de 15 a 19 años, este comportamiento de la variable contrasta con la creencia tradicional de la ayuda de los jóvenes en los oficios del hogar es principalmente de las niñas, puesto que los resultados de este trabajo señalan que la causa tiene un mayor efecto sobre los varones (Figura 17).

Figura 17.

\section{Desescolarizados por realización de oficios en el hogar según sexo y edad}

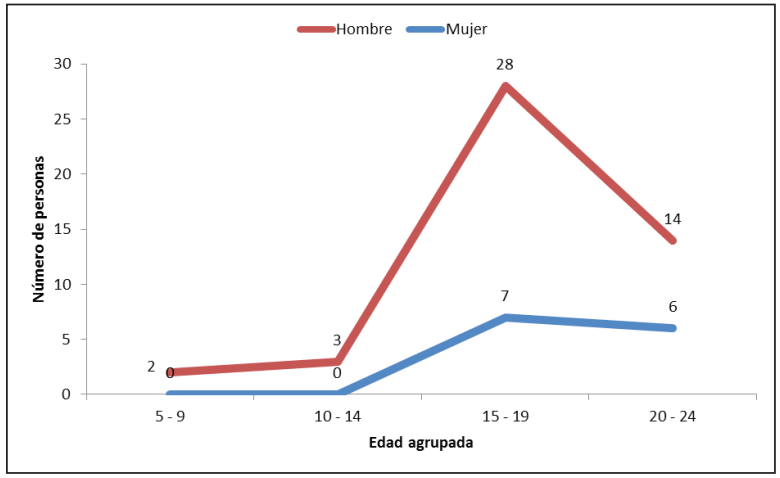

Fuente: Elaboración propia basado en la información del censo de desescolarizados.

Desescolarizados por razones y comunas: Para conocer la incidencia del fenómeno en las distintas comunas, se presenta el número de casos por las principales razones de desescolarización, para el caso de la comuna Santa Fé-Bastidas (comuna 5), Mamatoco-11 de Noviembre (comuna 6) y la comuna Parque-Bureche (comuna 9) se observa que el mayor número de casos se presentan por problemas en la casa (con 23, 159 y 44 casos respectivamente), señalando esta razón como la de mayor incidencia para estas zonas, pero para el caso específico de la comuna Pozos Colorados (comuna 8) la maternidad es la razón que mayor relevancia presenta con 50 casos, de este modo estos resultados permiten señalar que el problema del embarazo y la maternidad adolescente se presenta con mayor relevancia para este sector de la ciudad, y que es la segunda razón en importancia en las demás comunas (Tabla 3). 
Tabla 3.

Personas por razones de desescolarización en las comunas 5, 6, 8 y 9

\begin{tabular}{|c|c|c|c|c|}
\hline \multirow{2}{*}{ Razones } & \multicolumn{5}{|c|}{ Comuna } \\
\cline { 2 - 5 } & $\begin{array}{c}\text { Santa } \\
\text { Fé-Bastidas }\end{array}$ & $\begin{array}{c}\text { Mamatoco-11 de } \\
\text { Noviembre }\end{array}$ & $\begin{array}{c}\text { Pozos Colorados- } \\
\text { Don Jaca }\end{array}$ & $\begin{array}{c}\text { Parque- } \\
\text { Bureche }\end{array}$ \\
\hline Problemas en la casa & 23 & 159 & 31 & 44 \\
\hline \begin{tabular}{c} 
Necesidad de trabajar \\
\hline $\begin{array}{c}\text { Cambio de casa de la } \\
\text { familia }\end{array}$
\end{tabular}$\quad 10$ & 105 & 38 & 14 \\
\hline Maternidad o paternidad & 11 & 55 & 22 & 9 \\
\hline Perdida del año escolar & 6 & 118 & 50 & 21 \\
\hline Oficios del hogar & 1 & 44 & 5 & 5 \\
\hline Estudios suficientes & 8 & 32 & 23 & 7 \\
\hline
\end{tabular}

Fuente: Elaboración propia basado en la información del censo de desescolarizados.

Razones de la desescolarización de mayor incidencia por edad y sexo: a modo de resumen se presentan las razones de desescolarización de mayor incidencia y la relación con la edad y el sexo, este es un ejercicio conceptual en el que se incluyen en las columnas la edad en la que la razón presentó mayor influencia.

Es así como podemos observar que para los hombres las mayores razones de desescolarización en edades entre 15 y 19 años se encuentra en: i.) los problemas en la casa; ii.) la pérdida del año escolar y iii.) los oficios del hogar; en la edad entre los 20 y 24 años se observa: i.) la necesidad de trabajar y ii.) la paternidad. Para las mujeres en la edad entre 15 a 19 años las razones más importantes son: i.) la maternidad o el embarazo, ii.) la pérdida del año escolar y iii.) los oficios del hogar; en la edad entre 20 a 24 años los de mayor incidencia son: i.) los problemas en la casa y ii.) la necesidad de trabajar. En la edad entre 5 a 14 años no se presenta una mayor incidencia de las razones presentadas, característica relacionada con los mayores avances que se han tenido a nivel nacional y municipal por el aumento de la cobertura principalmente en los primeros años de estudio (Tabla 4).
Tabla 4.

\section{Razones de la desescolarización por edad de mayor incidencia (2011)}

\begin{tabular}{|c|c|c|}
\hline \multirow{2}{*}{ Razones } & \multicolumn{2}{|c|}{$\begin{array}{c}\text { Edad de mayor } \\
\text { incidencia de la } \\
\text { desescolarización }\end{array}$} \\
\cline { 2 - 3 } & Hombre & Mujer \\
\hline Problemas en la casa & $15-19$ & $20-24$ \\
\hline $\begin{array}{c}\text { Necesidad de trabajar } \\
\text { Embarazo, maternidad o } \\
\text { paternidad }\end{array}$ & $20-24$ & $20-24$ \\
\hline $\begin{array}{c}\text { Perdida de año escolar } \\
\text { Oficios del hogar }\end{array}$ & $15-19$ & $15-19-19$ \\
\hline
\end{tabular}

Fuente: Elaboración propia basado en la información del censo de desescolarizados. 


\section{Resultados del modelo logístico}

En este último punto se identificó la relación que existe entre las variables independientes y la posibilidad de ser desescolarizado (variable dependiente). Para realizar este ejercicio se utilizó la regresión logística, teniendo en cuenta que la variable que buscamos explicar es una variable dicotómica (con dos posibles respuestas: desescolarizado y no desescolarizado), explicada a través de otras variables, llamadas covariables (variables cuantitativas) y factores (las variables cualitativas) $)^{2}$.

Variables y coeficientes del modelo ${ }^{3}$ : en el modelo se encontraron dos variables significativas, estas son el sexo y la edad (Tabla 5).

Tabla 5.

\section{Variables en la ecuación}

\begin{tabular}{|c|c|c|c|c|c|c|c|}
\hline & & B & E.T. & Wald & gl & Sig. & $\operatorname{Exp}(B)$ \\
\hline \multirow{3}{*}{ Paso $3^{a}$} & $\operatorname{sexo}(1)$ & .357 & .055 & 42.889 & 1 & .000 & 1.430 \\
\hline & Edad & .122 & .005 & 561.051 & 1 & .000 & 1.130 \\
\hline & Constante & -3.734 & .095 & 1544.080 & 1 & .000 & .024 \\
\hline
\end{tabular}

a. Variable(s) introducida(s) en el paso 2: sexo.

La variable sexo nos muestra que para el caso de los hombres (1), se presentan 0,43 veces más posibilidades de ser desescolarizado, en comparación con las mujeres. La variable edad señala que por cada año más en una persona, se presentan 0,13 veces más posibilidades de ser desescolarizado.

Este modelo sirve como evidencia al patrón observado en la desescolarización por sexo y edad, que se presentó en la parte descriptiva de este documento, en donde se observó que de manera general los hombres tienen mayor incidencia a la desescolarización, así como también se ve un aumento de la incidencia cuando se da un aumento en la edad del individuo.

\section{Discusión}

El impacto de la política pública nacional en el aumento de cobertura en la primaria, secundaria y media, ha asegurado una mayor cobertura en todos los niveles, pero esta ha incidido principalmente en el nivel de primaria y los primeros años de secundaria; sin embargo, es necesario crear estrategias que permitan asegurar la educación de los jóvenes con edades entre 15 a 24 años, puesto que si se entiende la educación como un proceso formativo del individuo, si este no supera la fase final de la educación en el bachillerato, se podría pensar en un fracaso o "desvió escolar" como menciona Becker (1985), citado en Esterle (2005, p. 33); además del bajo nivel formativo del individuo y la disminución de las competencias para acceder a un trabajo formal, esta consideración surge teniendo en cuenta que es en la población de las comunas en edad de 15 a 24 años en donde la desescolarización presenta mayor incidencia, y es efecto de un contexto social, unas características marginales de los hogares y la valoración de la educación que hace que el individuo 
valore más trabajar en el momento, que invertir su dinero y su tiempo en educación, en donde recibirá beneficios solo hasta el final de la formación.

Al comparar las cifras del Censo de Población Nacional del año 2005 realizado por el DANE en Santa Marta con los resultados de esta investigación, se observa correspondencia en los dos ejercicios al señalar como una de las razones de desescolarización de mayor relevancia la razón "necesidad de trabajar", indicando la permanencia de este fenómeno a través del tiempo y su incidencia en las comunas analizadas, puesto que el censo suministra información del año 2005 para el Distrito, mientras el censo de desescolarización aplicado en este trabajo presenta resultados del año 2011 para las comunas seleccionadas.

Para el caso de la razón de desescolarización "embarazo", en el censo del 2005 representa el 2,9\%, en los resultados de este trabajo se agrupan embarazo, maternidad y paternidad que alcanza el $12 \%$, señalando que esta razón cobra importancia en las comunas analizadas en la ciudad de Santa Marta, principalmente en la comuna 8, donde el fenómeno presenta mayor incidencia en comparación con las otras comunas. Otra característica a detallar es que en la información censal del 2005 la edad en donde existe un mayor caso de mujeres desescolarizadas por embarazo es en la edad de 20 a 24 años, sin embargo los resultados encontrados para el área de estudio en Santa Marta señala que esta razón se manifiesta con mayor incidencia en las mujeres de edad entre 15 a 19 años, este cambio puede estar relacionado con que específicamente para esta área de estudio las mujeres están adelantando la edad de la maternidad y por ende su efecto en la desescolarización.

La variable "falta de cupos", aunque se presenta relevante para los datos censales de 2005, para los resultados del estudio esta razón no se presenta con importancia porcentual en comparación con las demás razones, esto puede ser el resultado de la política pública nacional y municipal enfocada al aumento de la cobertura.

La desescolarización se presenta con mayor incidencia para los grupos de edad entre 15 a 24 años, como consideración general de este trabajo se propone generar programas de impacto para la disminución de la desescolarización de ese grupo poblacional, y principalmente haciendo énfasis en las personas en edad entre 15 a 19 años, si se tiene en cuenta que esta población es la que debería estar en la educación secundaria y media, este trabajo señala pautas de acción para incidir en la disminución de la desescolarización de esas población basadas en las principales razones de desescolarización, de este modo se señala la necesidad de generar programas que incidan en la disminución de la pérdida del año escolar a través del acompañamiento académico, la discusión con el grupo familiar sobre la asignación de tareas u oficios en el hogar que causan efectos en la no asistencia a la escuela. Para el caso de las mujeres, la aplicación de programas enfocados a la prevención del embarazo adolescente, y en los hombres el dialogo familiar de los problemas en la casa que motivan la desescolarización. Es necesario realizar estudios con mayor profundidad de carácter interdisciplinario, con un alto componente psico-social y de trabajo social para conocer las manifestaciones sociales de las razones presentadas en este trabajo.

Por otra parte, se pueden observar en el Figura 2 las personas con edad mayor a 24 años y que no son bachilleres (5.785), que alcanzan el $45 \%$ del resto de la población, lo que resalta la necesidad de otro tipo de política pública o de programas tendientes a la culminación de los estudios del grupo poblacional con edad mayor de 24 años.

\section{Referencias Bibliográficas}

BANCO MUNDIAL. (1995). Priorities and strategies for education. Recuperado de CEPAL/OEA, de www.cepal.org: http://www. eclac.org/publicaciones/xml/2/4982/impact2.html

Cohen, E. (1997). Educación eficiencia y equidad: una difícil convivencia. Santiago de Chile, : Ediciones Sur, 1a. edición, p. 7. Recuperado de http://www.sitiosur.cl/publicacionescatalogodetalle.php?PID=2668\&doc $=Y \&$ lib $=Y \&$ rev $=Y \&$ art=Y\&doc1=Y\&vid=Y\&autor=\&coleccion=Colecci $\% F 3 n \% 20$ Estudios\%20Sociales\&tipo=ALL\&nunico=414\#descargar.

Constitución Política de Colombia. (1991). Recuperado de http://www.alcaldiabogota.gov.co/sisjur/normas/Norma1. jsp?i=4125. Artículo 67. 
DANE. (2012). Departamento Nacional de Estadistica. Recuperado de http://www.dane.gov.co/files/investigaciones/boletines/educacion/presentacion_EDUC_2011.pdf

Esterle, M. (2005). PREVENCIÓN Y TRATAMIENTO DEL ABSENTISMO Y DE LA DESESCOLARIZACIÓN EN FRANCIA: EXPERIENCIAS Y NUEVAS FORMAS DE ACTUAR. Revista Electrónica Iberoamericana sobre Calidad, Eficacia y Cambio en Educación, Vol. 3, Número 1, especial, 895-905. Recuperado de http://redalyc.uaemex.mx/pdf/55130182. pdf.

GOBIERNO VASCO. (2003). PROGRAMA PARA GARANTIZAR EL DERECHO A LA EDUCACIÓN: erradicación de la desescolarización y el absentismo escolar en el territorio de Biskaia . GOBIERNO VASCO.

LEY 375. (1997). Secretaria del Senado. Recuperado el 3 de Septiembre de 2012. Recuperado de http://www.secretariasenado.gov.co/senado/basedoc/ley/1997/ley_0375_1997. html
LEY GENERAL DE EDUCACIÓN. (1994). ALCALDÍA DE BOGOTA. Recuperado de http://www.alcaldiabogota.gov.co/sisjur/ normas/norma1.jsp?i=292

Ramírez, T., \& Téllez, J. (2006). La educación primaria y secundaria en Colombia en el siglo XX. Banco de la República.

SIGOT. (2005). SISTEMA DE INFORMACIÓN GEOGRÁFICA PARA LA PLANEACIÓN Y EL ORDENAMIENTO TERRITORIAL. Recuperado de http://sigotn.igac.gov.co/sigotn/

UNESCO, CEPAL. (2005). La educación como eje del desarrollo humano. En C. UNESCO, OBJETIVOS DE DESARROLLO DEL MILENIO: UNA MIRADA DESDE AMÉRICA LATINA Y EL CARIBE (págs. 83 - 110). Santiago de Chile. Recuperdado de www. cepal.org.

Weller, J. (2009,). LOS JÓVENES Y EL EMPLEO EN AMÉRICA LATINA DESAFÍOS Y PERSPECTIVAS ANTE EL NUEVO ESCENARIO LABORAL. Bogotá, Colombia: CEPAL, p. 15. Recuperado de www.cepal.org. 